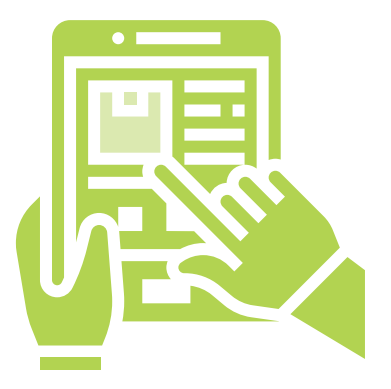

\title{
PRINCÍPIOS DE QUALIDADE BASEADOS EM HEURÍSTICAS DE USABILIDADE APLICADOS À FERRAMENTA RETRATOS DA ATENÇÃO PRIMÁRIA À SAÚDE
}

\author{
José Adailton da Silva \\ Doutor em Saúde Coletiva -Universidade Federal do Rio Grande do Norte - UFRN \\ Mestre em Saúde da Família - Rede Nordeste de Formação em Saúde da Família - RENASF/FIOCRUZ/ \\ UFRN. Professor Adjunto da Universidade Federal do Rio Grande do Norte - UFRN/FACIS. Coordenador \\ Pedagógico do Programa de Educação Permanente em Saúde da Família - PEPSUS/LAIS/SEDIS. \\ E-mail: joseadailtonmec@gmail.com
}

\section{Daniele Montenegro da Silva Barros}

Doutora e Mestre pelo Programa de Pós-Graduação em Engenharia Elétrica e Computação pela Universidade Federal do Rio Grande do Norte. Graduada em Tecnologia em Análise e Desenvolvimento de Sistemas pelo Instituto Federal de Educação, Ciência e Tecnologia do Rio Grande do Norte, Pesquisadora no Laboratório de Inovação Tecnológica em Saúde LAIS/UFRN.

E-mail:daniele.barros@lais.huol.ufrn.br

\section{Philippi Sedir Grilo de Morais}

Doutorando e Mestre em Engenharia Elétrica e Computação na Universidade Federal do Rio Grande do Norte. Graduado em Tecnologia em Análise e Desenvolvimento de Sistemas no IFRN - Instituto Federal de Educação, Ciência e Tecnologia do Rio Grande do Norte. Pesquisador no Laboratório de Inovação Tecnológica em Saúde LAIS/UFRN. E-mail: sedir.morais@lais.huol.ufrn.br

\section{Cesimar Xavier de Souza Dias}

Mestrado profissional em Engenharia de Software pela Universidade Federal do Rio Grande do Norte. Especialização em MBA em Marketing pela Universidade de São Paulo. Especialização em Avaliação do Ensino e da Aprendizagem pela Universidade do Oeste Paulista. Graduação em Curso Superior de Tecnologia em Design Gráfico pela Universidade Potiguar.

E-mail: cesimar.xavier@ifrn.edu.br

\section{Arthur Meireles da Silva}

Graduando em Análise e Desenvolvimento de Sistemas no Instituto Federal de Educação, Ciência e Tecnologia do Rio Grande do Norte. Pesquisador do Núcleo Avançado de Inovação Tecnológica.

E-mail: arthur.silva@navi.ifrn.edu.br 


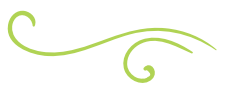

\section{RESUMO}

O desenvolvimento de sistemas focados no usuário vem se tornando cada vez mais relevante no contexto digital. Prover interfaces que reduzam a quantidade de trabalho e aumentem a capacidade de eficiência dos usuários é uma premissa cada vez mais latente. O presente trabalho objetivou melhorar a interface da versão anterior do sistema Retratos da Atenção Primária à Saúde com base nos objetivos primários da plataforma para o Programa Nacional de Melhoria do Acesso e da Qualidade da Atenção Básica.. Seu objetivo fim é atender à demanda do projeto de pesquisa desenvolvido pelo laboratório do Instituto Federal de Educação, Ciência e Tecnologia do Rio Grande do Norte, o Núcleo Avançado de Inovação Tecnológica e dar transparência aos dados relativos ao terceiro ciclo do Programa Nacional de Melhoria do Acesso e da Qualidade da Atenção Básica, do Governo Federal. Foi utilizado como suporte de qualidade de interface, no desenvolvimento do projeto, as heurísticas de usabilidade Nielsen e ergonomia para interfaces digitais. A melhoria da interface se deu gradativamente ao longo das etapas do projeto, com a inclusão de novas funcionalidades demandadas pela equipe da Coordenação Geral de Acompanhamento e Avaliação do Departamento de Atenção Básica do Ministério da Saúde.

PALAVRAS-CHAVE: Ergonomia. Heurísticas. Gestão em Saúde. Atenção Primária à Saúde. Usabilidade.

\section{ABSTRACT}

The development of user-centered systems has become increasingly relevant in the digital context. Providing interfaces that reduce the amount of work and increase users' efficiency is currently a latent premise. This paper addressed the improvement of the interface and its ergonomics for the web system Retratos da Atenção Primária à Saúde. Its main objective is to meet the demand of the research project developed by the Núcleo Avançado de Inovação Tecnológica (NAVI) and to provide transparency to the data related to the Programa Nacional de Melhoria do Acesso e da Qualidade da Atenção Básica (PMAQ-AB) of the Federal Government. Nielsen's usability heuristics and ergonomics for digital interfaces were used to support the quality of the project. The improvement of the interface took place gradually throughout the stages of the project, with the inclusion of new features demanded by the General Coordination of Monitoring and Evaluation, related to the Department of Primary Care of the Ministry of Health.

KEY WORDS: Ergonomics. Heuristics. PMAQ-AB. Retratos da Atenção Primária à Saúde. Usability. 


\section{INTRODUÇÃO}

O Programa Nacional de Melhoria do Acesso e da Qualidade da Atenção Básica (PMAQ-AB) foi instituído pelo Ministério da Saúde com o objetivo de melhorar processos que ampliassem a capacidade da gestão federal, estadual e municipal, bem como a eficácia nos serviços de cuidados prestados, pelas equipes de saúde, à população no território da Atenção Primária à Saúde (APS) (Portaria GM/MS n 1.654, 2011). O PMAQ-AB foi criado em 19 de julho de 2011, sendo estruturado em três fases para assegurar a melhoria do acesso e da qualidade aos serviços da atenção primária, sendo estas: Adesão e contratualização, Certificação e Recontratualização (BRASIL, 2017).

O PMAQ-AB tem um papel de relevância no progresso da APS nacional e, conforme a Portaria-GM/MS 1.645/2015, propõe um conjunto de estratégias de qualificação, acompanhamento e avaliação do trabaIho das equipes de saúde, como também determina a transparência em todas as suas etapas, de modo a permitir o contínuo acompanhamento de suas ações e resultados pela sociedade (BRASIL, 2015).

No entanto, apesar de fazer parte de suas diretrizes, a transparência do programa se apresentou falha, fazendo com que no ano de 2017, o Tribunal de Contas da União (TCU) apontasse ao Ministério da Saúde, que os dados do programa não foram divulgados ao público geral até a data de publicação do acórdão (Acórdão n²019/2017-PL). No escopo textual, o TCU se refere aos dados da avaliação externa realizada no $2^{\circ}$ ciclo do programa, o qual foi finalizado em junho de 2015.

Destaca-se que a transparência é indispensável na gestão pública e, em se tratando do PMAB-AB é basilar para o planejamento das ações pelos gestores e profissionais das equipes, que podem analisar os seus indicadores e traçar novas estratégias de melhoria da qualidade.

Partindo do princípio da transparência, o Ministério da Saúde, por meio do Termo de Execução Descentralizada (TED) n'114/2018, pactuou o desenvolvimento de sistemas, dentre eles o Retratos da Atenção Primária à Saúde - uma plataforma que apresenta os dados oriundos das avaliações sobre o acesso e a qualidade das equipes de saúde participantes do PMAQ-AB em todo o país. Com sua implementação, foi possível dar acesso aos dados à sociedade, sejam estes agentes do Sistema Único de Saúde (SUS), sejam gestores de todas as esferas, pesquisadores ou público geral.

Assim, este trabalho objetivou melhorar a interface da versão anterior do sistema Retratos da Atenção Primária à Saúde com base nos objetivos primários da plataforma para o Programa Nacional de Melhoria do Acesso e da Qualidade da Atenção Básica.

\section{OS PRINCÍPIOS DE QUALIDADE A PARTIR DAS HEURÍSTICAS DE USABILIDADE}

Trata-se de uma pesquisa aplicada, intervencionista, para a melhoria no processo de interação de usuários na ferramenta, por meio da alteração da interface da versão antecessora. Essa alteração foi norteada pela perspectiva do design centrado no usuário - DCU, considerando que a leitura dos dados deve ser realizada por múltiplos perfis de usuários utilizadores. Cada usuário tem um modo diferente de resolver os problemas (LOWDERMILK, 2013) e, por isso, o desenvolvimento centrado no usuário se torna uma metodologia pertinente para projetos com alto grau de usabilidade.

Para Benyon (2011), um sistema com alto grau de usabilidade terá as seguintes características: será eficiente, com uma quantidade adequada de esforço; será eficaz, no sentido de que conterá funções e conteúdos 
organizados de forma apropriada; será fácil de aprender; será seguro de utilizar, mesmo diante de diversos contextos distintos; terá alto grau de utilidade, considerando que a interface não romperá as perspectivas dos usuários. Nielsen (1993) explica que a usabilidade é um atributo de qualidade que determina o quão fácil é uma interface. Este autor (NIELSEN, 2012) defende que uma interface com usabilidade deve conter minimamente as seguintes características: fácil de manusear; simples de aprender; ter relações com os modelos mentais dos usuários, sendo difícil de esquecer; prevenir erros do usuário; ser eficiente no uso.

Além disso, do ponto de vista do desenvolvimento de software, para ampliar a capacidade de desenvolvimento e incorporar mais qualidade dos produtos criados, lançar mão de padrões de interface web pode conferir melhoria não somente no projeto, mas também nos resultados. Dias (2019) apresenta três características que validam isso ao explicar que a adoção de padrões de interface permite: (i) minimizar o tempo e esforço de design; (ii) aumentar a qualidade das soluções de design e (iii) auxiliar na comunicação entre os designers e programadores.

Nessa perspectiva, o uso de princípios testados e aprovados que priorizaram o usuário como elemento para a criação de produtos interativos melhoram e resultam em projetos com alta aceitabilidade (PREECE; ROGERS; SHARP, 2002). A usabilidade busca melhorar não somente a interface de software, mas também a interação, convergindo com as perspectivas do usuário. Por isso, o desenvolvimento centrado no usuário busca entender quem são os usuários utilizadores, suas expectativas, experiências e o escopo envolvido para desenvolver projetos eficazes.

Experiências anteriores e práticas que são comumente repetidas geram expectativas futuras aos usuários. Isso faz com que os usuários consigam ter melhor desempenho quando seus modelos mentais são respeitados e as interfaces refletem esses modelos (HARLEY, 2017). Dias (2019) afirma que quando não se respeita os padrões a que os utilizadores já estão habituados, há uma ruptura nas expectativas deles, fazendo com que estes precisem reaprender uma interface que deveria estender os padrões estabelecidos ao longo da sua trajetória cognitiva.

Neste contexto, a utilização das Heurísticas de Usabilidade se tornou extremamente relevante. A elaboração da nova interface buscou alcançar as seguintes metas de usabilidade: eficácia, eficiência, facilidade de memorização e facilidade de aprendizado (CYBIS; BETIOL; FAUST, 2015). E utilizou as seguintes heurísticas de usabilidade: design estético e minimalista, consistência de padrões, controle do usuário e liberdade, e flexibilidade, reconhecimento em vez de memorização e, por fim, eficiência de uso. As demais heurísticas ficaram fora do escopo do projeto, considerando os objetivos primários e tempo disponível para finalização do projeto.

\section{TECNOLOGIAS E FERRAMENTAS UTILIZADAS NO DESENVOLVIMENTO DA INTERFACE}

Para o desenvolvimento da interface do sistema foram utilizadas as seguintes tecnologias: (i) a linguagem de marcação HTML (Hypertext Markup Language), em sua versão 5; (ii) a linguagem de estilo CSS (Cascading Style Sheet) com as características mais atualizadas da versão 3; (iii) o framework Bootstrap, focado em dar suporte básico nos componentes reaproveitáveis; (iv) a linguagem Javascript e o framework Vue JS nas páginas a partir de componentes adaptáveis e reutilizáveis em diferentes partes do sistema. Assim como o Vue JS, foram utilizadas outras duas ferramentas de terceiros: highcharts, um projeto baseado em Javascript que tem a finalidade 
de gerar gráficos; e datatables uma biblioteca Javascript utilizada para gerenciar a apresentação dos dados por meio de tabelas.

\section{INTERFACES E FUNCIONALIDADES DO SISTEMA}

O sistema dispõe de quatro diferentes tipos de consulta aos dados do PMAQ-AB. As funcionalidades foram projetadas para que os diferentes perfis de usuários pudessem ter liberdade para consultar e construir as informações do PMAQ-AB com uma visualização simples, proporcionada através do uso de gráficos. O Quadro 1 apresenta as diferenças entre os tipos de busca.

Quadro 1 - Tipos de buscas presentes na interface do sistema Retratos.

\begin{tabular}{|l|l|}
\hline \multicolumn{1}{|c|}{ Tipo de busca } & \multicolumn{1}{c|}{ Perfil de usuário } \\
\hline Busca por temática & $\begin{array}{l}\text { Usuários com baixo conhecimento } \\
\text { sobre a ferramenta. }\end{array}$ \\
\hline Busca avançada & $\begin{array}{l}\text { Usuários que conhecem as variáveis } \\
\text { do instrumento PMAQ-AB. }\end{array}$ \\
\hline $\begin{array}{l}\text { Consulta de } \\
\text { Eertificação das }\end{array}$ & $\begin{array}{l}\text { Usuários que buscam informações } \\
\text { sobre a qualidade das equipes } \\
\text { participantes do terceiro ciclo do } \\
\text { programa. }\end{array}$ \\
\hline $\begin{array}{l}\text { Cruzamento de } \\
\text { Variáveis }\end{array}$ & $\begin{array}{l}\text { Usuários com expertise para buscar } \\
\text { relações entre dados existentes no } \\
\text { instrumento PMAQ-AB por meio do } \\
\text { cruzamento de variáveis. }\end{array}$ \\
\hline
\end{tabular}

Fonte: Elaborado pelos autores (2020).

\section{Busca por Temática}

A Busca por Temática é uma funcionalidade projetada para que usuários sem o domínio sobre o instrumento possam acessar as informações desejadas sem impedimentos. Por meio de uma série de filtros, o usuário é levado a um gráfico e a um mapa que apresenta os resultados da consulta. O fluxo da funcionalidade sofreu mudanças na versão. Anteriormente era disposto um padrão de menu, em formato de mapa de site, que se expandia de acordo com a opção selecionada (Figura 1).

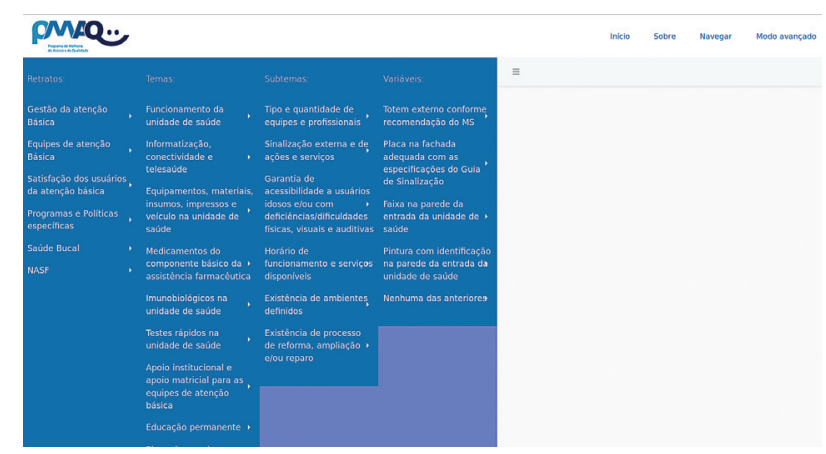

Figura 1 - Forma de acesso via menu da versão anterior da Busca por Temática.

Fonte: Retratos da Atenção Primária à Saúde (2020).

Após selecionar a região desejada, o usuário deve escolher o retrato, tema, subtema e variável de pesquisa. Isto, na forma como ocorreu, implicou um problema grave de acesso às informações, por dispô-las exigindo rolagem excessiva da tela e aumentando as chances de erro ao recorrer a uma opção indesejada. Para correção deste item, foi realizada uma readequação do acesso através das heurísticas de reconhecimento em vez de memorização e equivalência entre sistema e mundo real.

Aqui os princípios se aplicam em uma estrutura de passos lógicos que conduzem o usuário a seu objetivo pautado na facilidade de reconhecer o caminho e na condução através de metáforas comuns ao seu modelo mental.

problema, portanto, foi solucionado na versão atual da interface, pois há, uma disposição da funcionalidade em formato de card $^{1}$, mais visível e organizado

\footnotetext{
${ }^{1}$ Constitui um grupo de padrões de interface do tipo conteúdo. São utilizados para exibir conteúdos com muitas informações.
} 
(Figura 2), que possibilita com a ação de um clique no elemento a visualização das opçães de localidade.

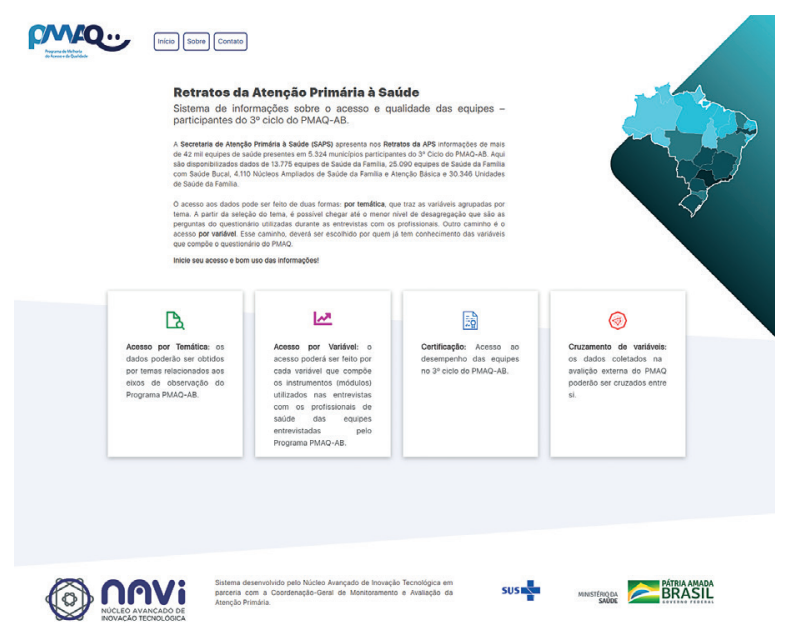

Figura 2 - Forma de acesso via card da versão atual da Busca por Temática.

Fonte: Retratos da Atenção Primária à Saúde (2020).

Cada região geográfica apresenta seu nome em ordem alfabética e uma imagem representativa, aparecendo abaixo dos cards de consulta, como exposto na Figura 3.

Figura 3 - Exibição das áreas geográficas.

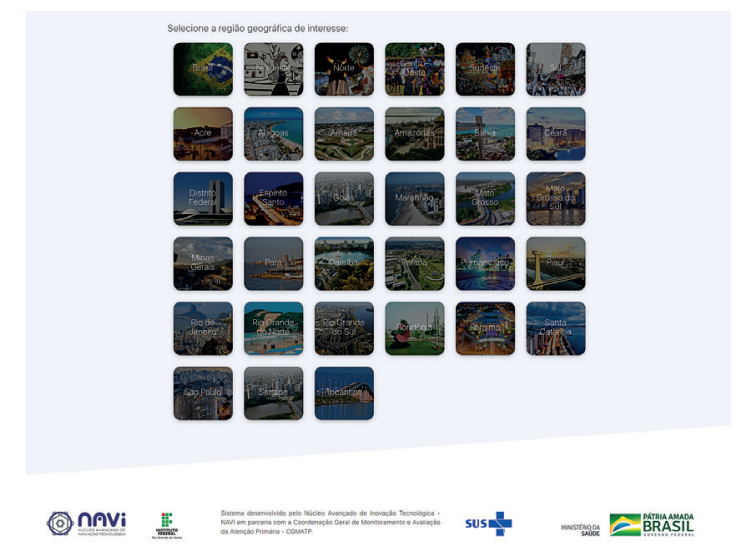

Fonte: Retratos da Atenção Primária à Saúde (2020).

Assim é possível escolher o retrato e depois selecionar o tema, subtema e variável de interesse nos filtros da aplicação. Na Figura 4, apresenta-se uma mudança geral na característica da ação de consulta a um dado, na forma de acesso por temática.

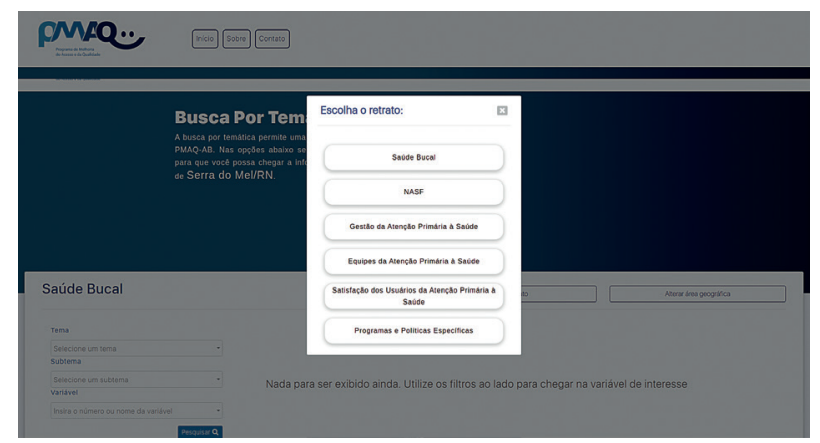

Figura 4 - Exibição dos retratos.

Fonte: Retratos da Atenção Primária à Saúde (2020). 
Outro problema da interface antiga era a impossibilidade de voltar às ações anteriores, refazê-las por meio de atalhos, ou ainda, analisar dois resultados, cujo processo de escolha tenha sido semelhante. Uma situação hipotética é de que o mesmo usuário procurou por uma variável no Retrato do Núcleo de Apoio à Saúde da Família (NASF) e, no entanto, não pôde facilmente procurar por outra variável relativa ao mesmo retrato. No fluxo anterior da interface, haveria de se refazer todo o conjunto de ações elencadas.

No entanto, essa dificuldade foi corrigida após a inclusão de atalhos, como "alterar região", "alterar retrato", ou ainda, a disposição do filtro de consulta ao lado do mapa (Figura 5). Para tanto, adotou-se, como base para o critério de qualidade do acesso, a heurística de Controle do Usuário e Liberdade, que prevê a saída de um estado indesejado de maneira simples.

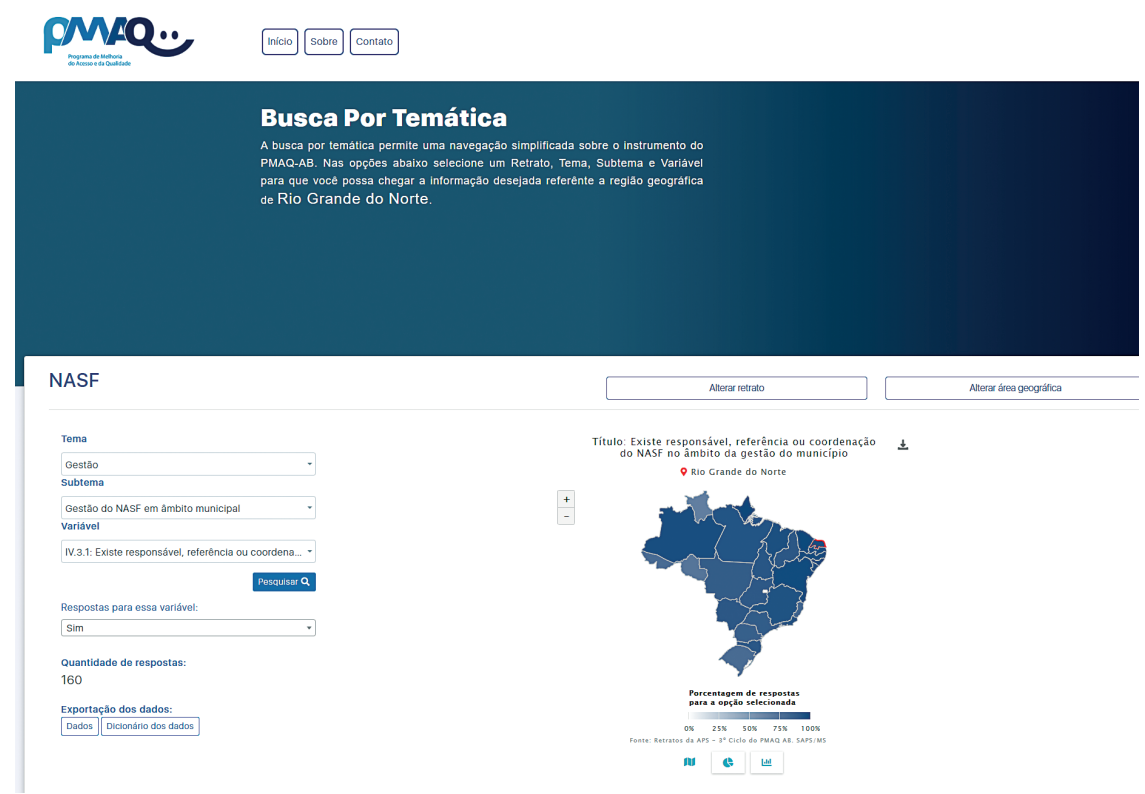

Figura 5 - Interface final da Busca por Temática.

Fonte: Retratos da Atenção Primária à Saúde (2020).

\section{Busca Avançada}

A busca avançada, diferentemente da funcionalidade anterior, é uma forma mais direta de se chegar aos dados desejados. Contudo, são necessários dois parâmetros mandatórios, são eles: região, que pode ser o país (Brasil), um estado ou município; e um termo de busca com no mínimo três caracteres que estejam contidos no nome ou conteúdo da variável buscada. Após isso, o sistema realiza uma busca de forma assíncrona com uma lista de variáveis em uma caixa de seleção (select) onde é possível escolher a variável desejada.

Na primeira versão da busca avançada, apresentada na Figura 6, é possível observar um layout distinto da interface apresentada na Figura 7. Essas mudanças foram feitas baseadas na heurística de Estética e Minimalismo, pois procurou-se dispor os elementos numa estrutura coesa e objetiva que apresentasse as informações a respeito do projeto e as variáveis de pesquisa. Isto deu um tom de clareza e trouxe facilidade ao acesso das funcionalidades. Além disso, a heurística de reconhecimento em vez de memorização foi usada para minimizar a carga de esforço mental na leitura dos dados, portanto, utilizando o mapa do Brasil; objeto de conhecimento popular e de fácil memorização. 

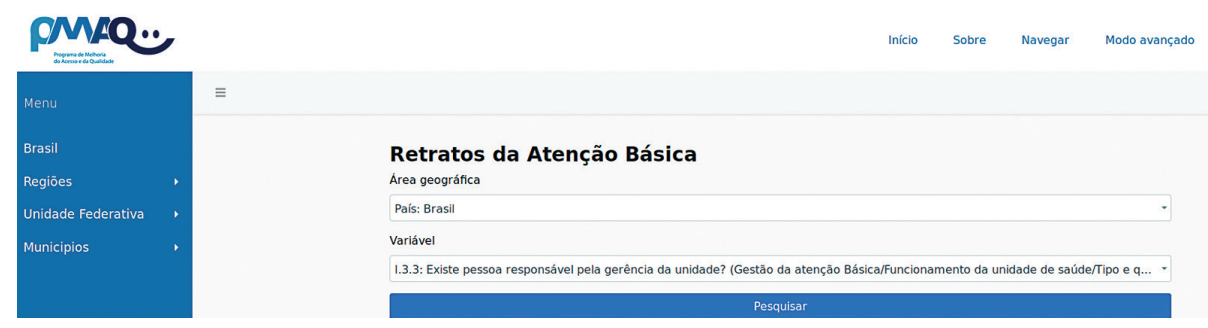

Figura 6 - Interface da busca avançada da versão anterior.

Fonte: Retratos da Atenção Primária à Saúde (2020).

\section{PMAQ.}

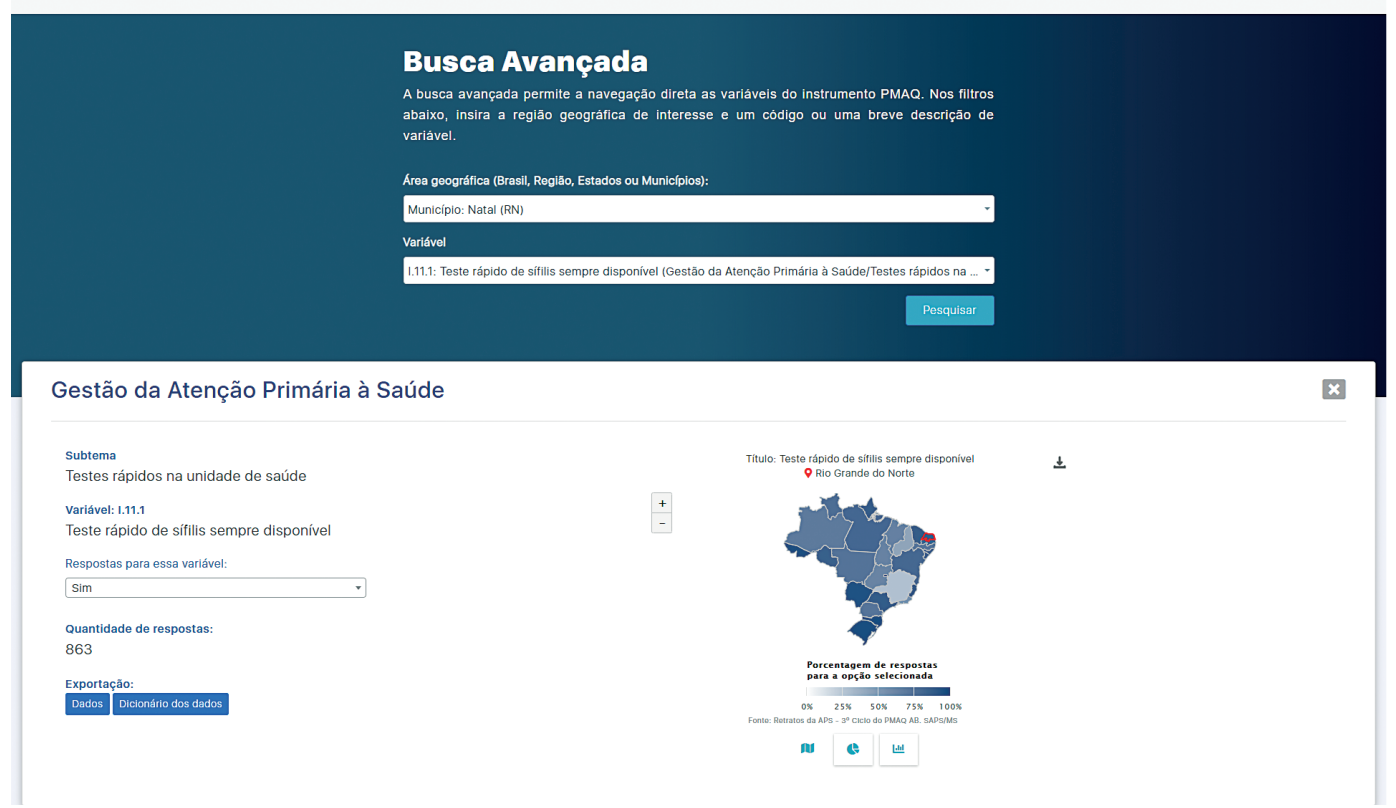

Figura 7 - Interface da busca avançada após mudança aplicando as heurísticas.

Fonte: Retratos da Atenção Primária à Saúde (2020).

\section{Área de certificação}

A área de certificação é uma funcionalidade que, por meio de um mapa interativo e uma tabela, possibilita as consultas do desempenho das equipes participantes em cinco categorias, as quais refletem o cumprimento de padrões de qualidade relacionados à oferta e organização da Atenção Primária à Saúde no Brasil. A área de certificação também permite a comparação da classificação das equipes de diferentes regiões.

Com relação à interface, as mudanças foram poucas e pontuais, também houve uma mudança nos requisitos. No início, a área de certificação apresentava duas abas e com a adesão da funcionalidade de comparação, foi adicionada uma nova aba que trouxe um gráfico em formato de barra com uma caixa de seleção (select) disponível para a inclusão de novas regiões para comparação. A Figura 8 apresenta a versão anterior dessa tela ao passo que a Figura 9 representa a versão final da interface da Área de Certificação. 


\section{PMAQ.-}
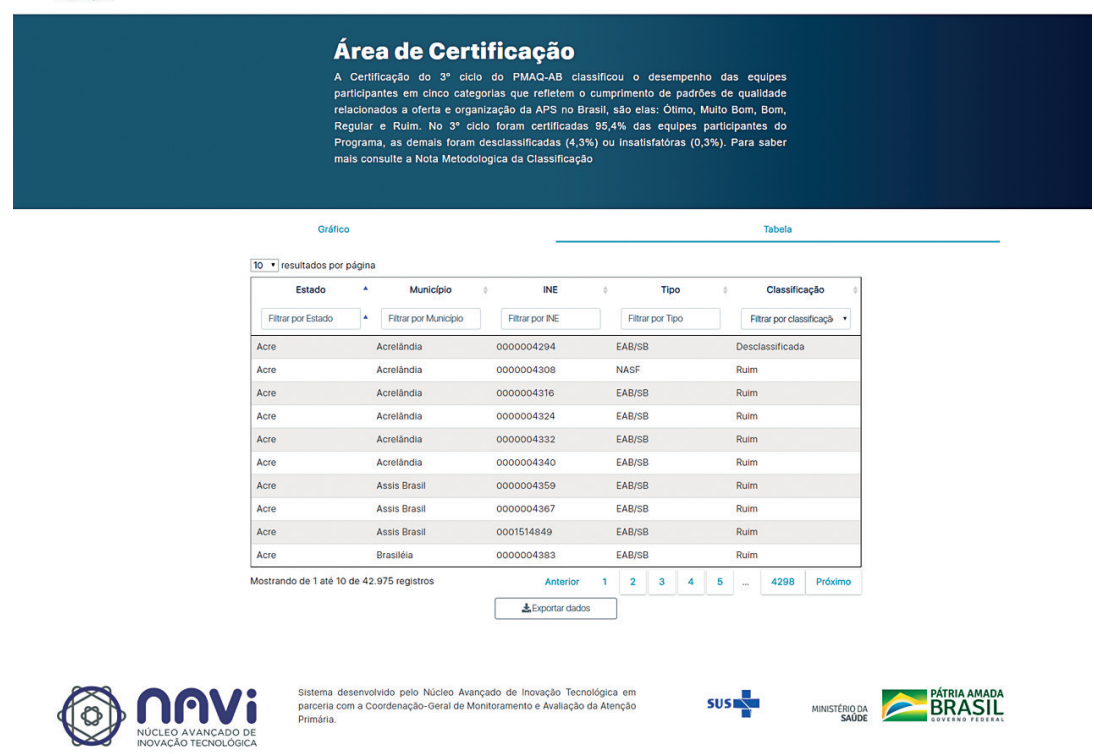

Figura 8 - Primeira versão da área de certificação.

Fonte: Retratos da Atenção Primária à Saúde (2020).

\section{PMAQ.}

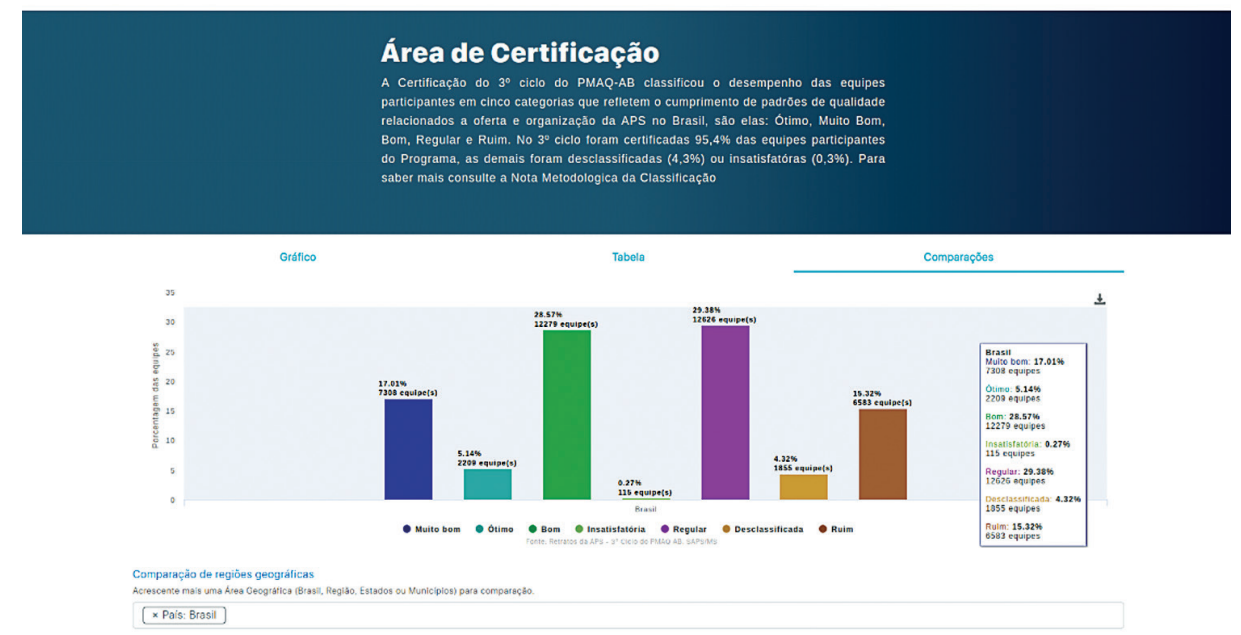

กคVi

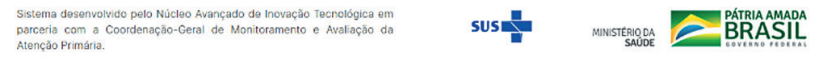

Figura 9 - Versão final da área de certificação.

Fonte: Retratos da Atenção Primária à Saúde (2020).

Nesta etapa, o redesign dos dois primeiros tipos de busca serviu como a base sólida para implementação dessa nova demanda da área de certificação, bem como de cruzamento de variáveis. Por essa razão, usou-se da orientação das heurísticas anteriores e seguiu-se a proposta dos critérios de qualidade já adotados. Para este caso da área de certificação, a adição de um mapa com os números da classificação da qualidade das equipes foi imprescindível, pois apresentou de forma direta às pessoas a informação requerida. 


\section{Cruzamento de variáveis}

O cruzamento de variáveis é uma funcionalidade que permite ao usuário, após selecionar duas variáveis e uma ou mais alternativas da variável, gerar uma tabela cruzando esses dados e um gráfico de barra comparando os resultados das duas variáveis selecionadas.

O layout da página de cruzamento de variáveis inicialmente era apenas um conjunto de caixas de seleção (selects), após uma avaliação foram realizadas alterações para dar destaque, de forma organizada. Essa mudança foi adotada com base na heurística de Estética e Minimalismo, através da qual objetivou-se a interação homem-interface de forma mais amigável que sua versão inicial (Figura 10). O que, posteriormente, pode implicar ganhos na eficácia e eficiência da consulta aos dados.

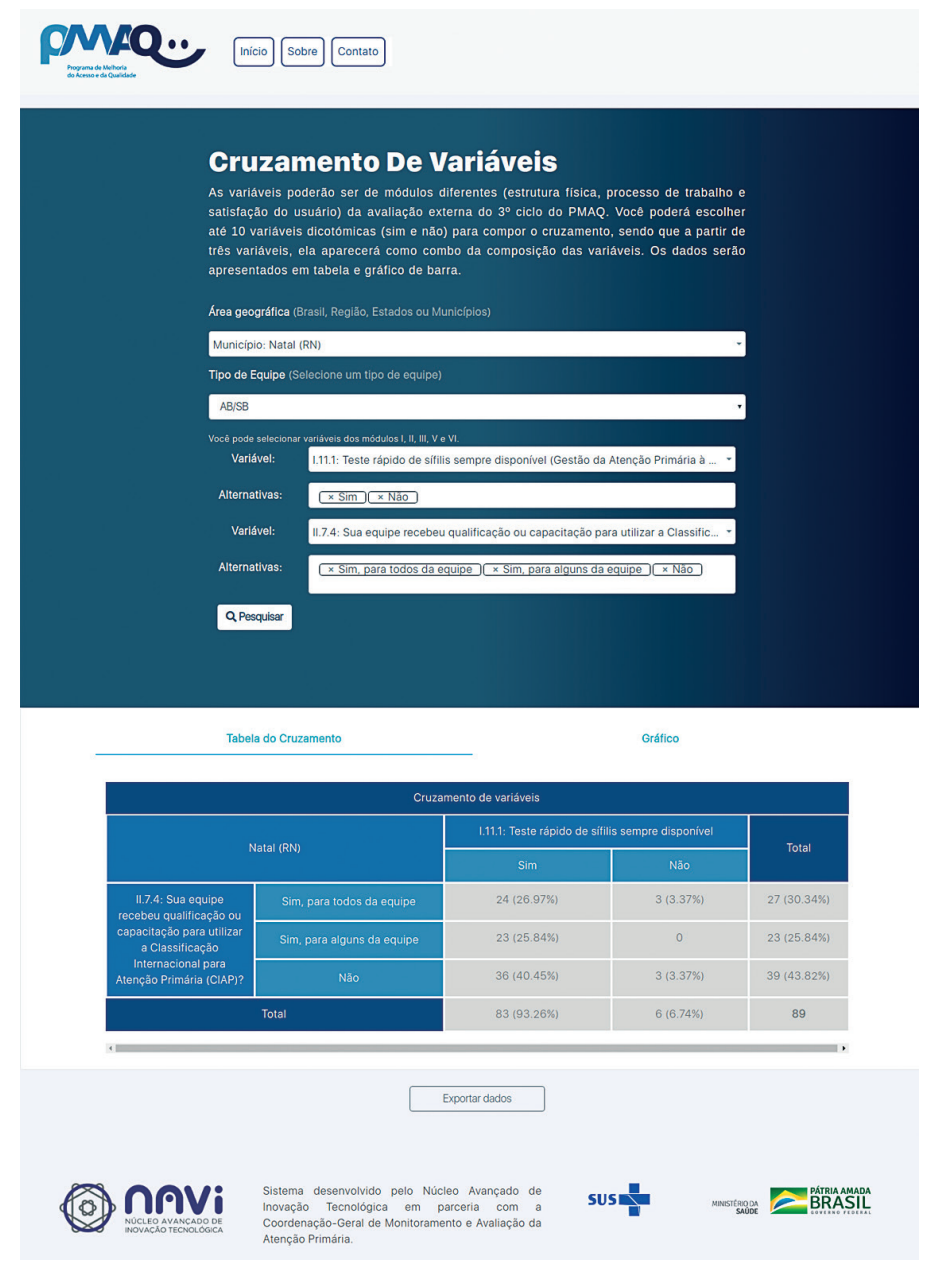

Figura 10 - Primeira versão do cruzamento de variáveis. Fonte: Retratos da Atenção Primária à Saúde (2020).

Neste projeto, o processo de avaliação heurística ocorreu de forma contínua. Por isso, após a implementação de cada versão, como a primeira dessas funcionalidades, foram realizadas novas análises e verificada a possibilidade de sua melhoria. Além das heurísticas anteriormente aplicadas, na versão final, foi aplicada a heurística de Estética e Minimalismo para a concatenação dos dados baseando-se em padrões visuais de interface. A aproximação dos elementos, trazendo a ideia de junção dos elementos comuns, dispostos na versão final da página do cruzamento de variáveis, é apresentado na Figura 11. 


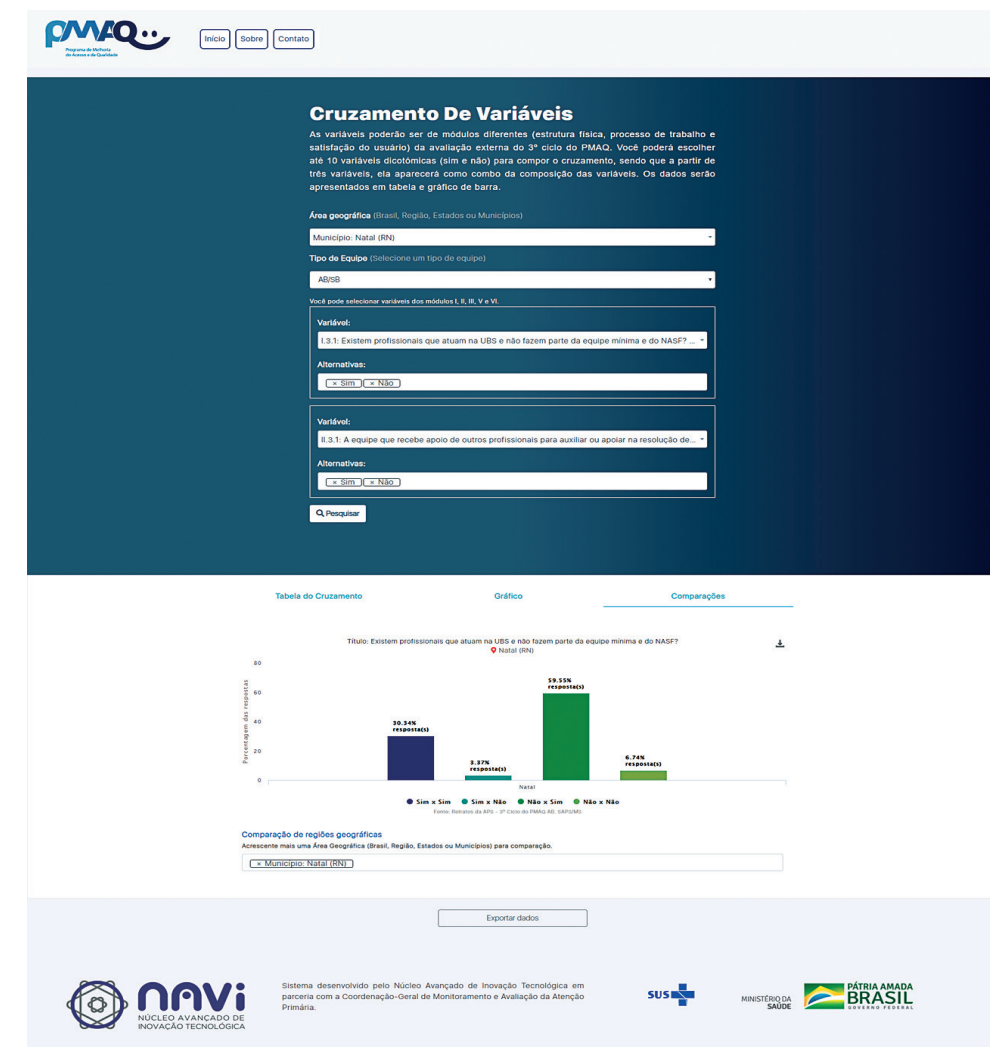

Figura 11 - Versão final do cruzamento de variáveis. Fonte: Retratos da Atenção Primária à Saúde (2020).

\section{CONCLUSÃO}

O objetivo deste trabalho foi melhorar a interface da versão anterior do sistema Retratos da Atenção Primária à Saúde com base nos objetivos primários da plataforma para o Programa Nacional de Melhoria do Acesso e da Qualidade da Atenção Básica. Esses objetivos foram demandados pela necessidade de melhoria do acesso aos dados para que diferentes perfis de usuários fossem capazes de explorá-los baseando-se nos seus próprios modelos mentais. A execução das alterações foi norteada pelos critérios padronizados das heurísticas de usabilidade, que se mostraram eficazes para melhorar a interface utilizando conhecimento amplamente testado e com custo de aplicação baixo, do ponto de vista de trabalho despendido pela equipe de software. Apesar da interface já ter sido construída anteriormente e refeita a partir das necessidades de alteração apontadas pela avaliação heurística realizada anteriormente, as mudanças conferiram mais qualidade na visibilidade e disponibilização dos dados. Além disso, o desenvolvimento deste trabalho contribuiu para que a plataforma cumprisse com a diretriz estabelecida no inciso III do artigo $3^{\circ}$ da portaria $N^{\circ} 1.645$ de 2 de outubro de 2015. No entanto, pelo fato do foco deste trabalho estar voltado para aplicação das heurísticas, comparando com a versão anterior, não foi possível, ainda, a aplicação de avaliação que possa aferir que as mudanças cumpriram o papel de melhoria na interação entre os usuários e a ferramenta. Para um trabalho futuro, será conduzida a avaliação com usuários que estão utilizando essa nova versão e assim sendo possível comparar os avanços entre a versão anterior e a atual. 


\section{REFERÊNCIAS}

BENYON, David. Interação Humano-Computador. 2. ed. São Paulo: Pearson Prentice Hall, 2011.

BRASIL. Ministério da Saúde. Extrato do Termo de Execução Descentralizada de Recursos $n^{\circ}$ 114/2018. Disponível em: https:// www.in.gov.br/materia/-/asset_publisher/Kujrw0TZC2Mb/content/ id/52017767. Acesso em: 19 out. 2020.

BRASIL. Ministério da Saúde. Portaria n 1.654, de 19 de julho de 2011. Institui, no âmbito do Sistema Único de Saúde, o Programa Nacional de Melhoria do Acesso e da Qualidade da Atenção Básica (PMAQ-AB) e o Incentivo Financeiro do PMAQ-AB, denominado Componente de Qualidade do Piso de Atenção Básica Variável - PAB Variável. Disponível em: http://bvsms.saude.gov.br/bvs/saudelegis/ gm/2011/prt1654_19_07_2011.html. Acesso em: 19 out. 2020.

BRASIL. Ministério da Saúde. Manual instrutivo para as equipes de atenção básica e NASF. Brasília: Ministério da Saúde, 2017.

BRASIL. Portaria $\mathbf{n}^{\circ}$ 1645, de 2 outubro de 2015. Dispõe sobre o Programa Nacional de Melhoria do Acesso e da Qualidade da Atenção Básica (PMAQ-AB). Disponível em: http://bvsms.saude.gov. br/bvs/saudelegis/gm/2015/prt1645_01_10_2015.html. Acesso em: 19 out. 2020.

BRASIL. Tribunal de Contas da União. Acórdão n 2019/2017. Plenário. Relator: Ministro Bruno Dantas. Sessão de 13/09/2017. Diário Oficial da União, Brasília, DF, 29 set. 2017. 2019/2017 PLENÁRIO: 030.300/2016-9. Relator: Bruno Dantas. DS: 13/09/2017.

CYBIS, W.; BETIOL, A.; FAUST, R. Ergonomia e Usabilidade: Conhecimentos, Métodos e Aplicações. 3. ed. São Paulo: Novatec Editora, 2015.

DIAS, C. X. de S. Smart Node Dashboard: um framework front-end baseado em Node-RED para criação de City Dashboards. 2019. 70 f. Dissertação (Mestrado) - Curso de Engenharia de Software, Instituto Metrópole Digital, Universidade Federal do Rio Grande do Norte, Natal, 2019.

HARLEY, A. Variations on Practiced Patterns Cause Mistakes. 2017. Disponível em: https://www.nngroup.com/articles/practiced-patternsmistakes/. Acesso em: 21 out. 2020. 
LOWDERMILK, T. Design centrado no usuário: um guia para o desenvolvimento de aplicativos amigáveis. São Paulo: Novatec, 2013.

NIELSEN, J. Usability 101: Introduction to Usability. 2012. Disponível em: https://www.nngroup.com/articles/usability-101-introduction-tousability/. Acesso em: 21 out. 2020.

NIELSEN, J. Usability engineering. San Diego: Morgan Kaufmann, 1993. $362 \mathrm{p}$.

NÚCLEO AVANÇADO DE INOVAÇÃO TECNOLÓGICA. Retratos da Atenção Primária à Saúde, 2020. Disponível em: https://retratos. navi.ifrn.edu.br. Acesso em: 15 out. 2020.

PREECE, J.; ROGERS, Y.; SHARP, H. Design de Interação: Além da interação homem-computador. Porto Alegre: Artmed, 2002. 